

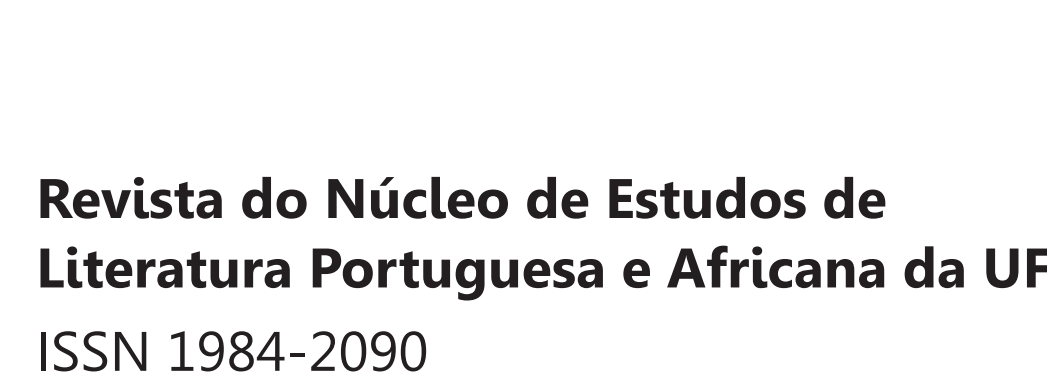

$$
\text { ABPIL }
$$


ABPIL - Revista do Núcleo de Estudos de Literatura Portuguesa e Africana da UFF Vol. 10, n²1, novembro de 2018

\section{EDITOR RESPONSÁVEL}

Ida Alves

Silvio Renato Jorge

\section{COMISSÃO EXECUTIVA}

Dalva Calvão

Iris Amâncio

Luis Maffei

Maria Lúcia Wiltshire de Oliveira

Renata Flavia da Silva

Tatiana Pequeno

\section{CONSELHO EDITORIAL}

Laura Cavalcante Padilha (UFF)

Ana Mafalda Leite (Universidade de Lisboa)

Benjamin Abdala Júnior (USP)

Cleonice Berardinelli (UFRJ)

Inocência Mata (Universidade de Lisboa)

João Barrento (Universidade. Nova de Lisboa)

Jorge Fernandes da Silveira (UFRJ)

Lelia Parreira Duarte (PUC-MG)

Margarida Calafate Ribeiro (CES/Universidade de Coimbra)

Maria Theresa Abelha (UFRJ)

Mário César Lugarinho (USP)

Maria Nazareth Soares da Fonseca (PUC-MG)

Monica Figueiredo (UFRJ)

Nuno Júdice (Universidade Nova de Lisboa)

Paulo Motta de Oliveira (USP)

Rosa Martelo (Universidade do Porto)

Silvio Renato Jorge (UFF)

\section{EQUIPE TÉCNICA}

Paloma Roriz, Revisora, UFF/CNPq, Brasil

Aline Duque Erthal, Revisora, UFF/Capes, Brasil

Elir Ferrari, Apoio técnico à editoração no SEER/OJS, UFF/UERJ, Brasil

Ivan Takashi Kano, Revisor, UFF, Brasil

Joana Lima, Laboratório de Livre Criação | UFF - IACS, Brasil

"Apoiado pela Universidade Federal Fluminense com recursos do Programa Auxílio Publicação - PROPPI, 2014"

\section{Dados para Catalogação:}

ABPYL - Revista do Núcleo de Estudos de Literatura Portuguesa e Africana da UFF. Niterói: NEPA/UFF, Vol. 10, n²1, novembro de 2018. Semestral.

Disponível em: http://www.revistaabril.uff.br/

\section{Periódicos. 1. Literatura Portuguesa;}

2. Literaturas Africanas de Língua Portuguesa;

3. Literatura Comparada. II. Literaturas de Língua Portuguesa: Teoria e Crítica. ISSN 1984-2090

\section{CORRESPONDÊNCIA}

NEPA - Revista Abril

Universidade Federal Fluminense - UFF | Instituto de Letras

Rua Prof. Marcos Waldemar de Freitas Reis, s/nº, sala 403, Bloco C - Campus do Gragoatá

São Domingos - Niterói - RJ | CEP 24210-201

Fone: (21)26292549 | 2629-2618 | 2629-2608 | E-mail: revistaabril@vm.uff.br

Projeto Gráfico: Diego Marques e Diagramação: Joana Lima

Capa: Cópia do mapa-múndi de Al-Idrissi, de 1154, feita em 1456 por Al-Qâsimî's, e que está na Bodleian Library, da Universidade de Oxford. Public Domain, https://commons.wikimedia.org/w/index.php?curid=101284. 
Revista do Núcleo de Estudos de Literatura Portuguesa e Africana da UFF

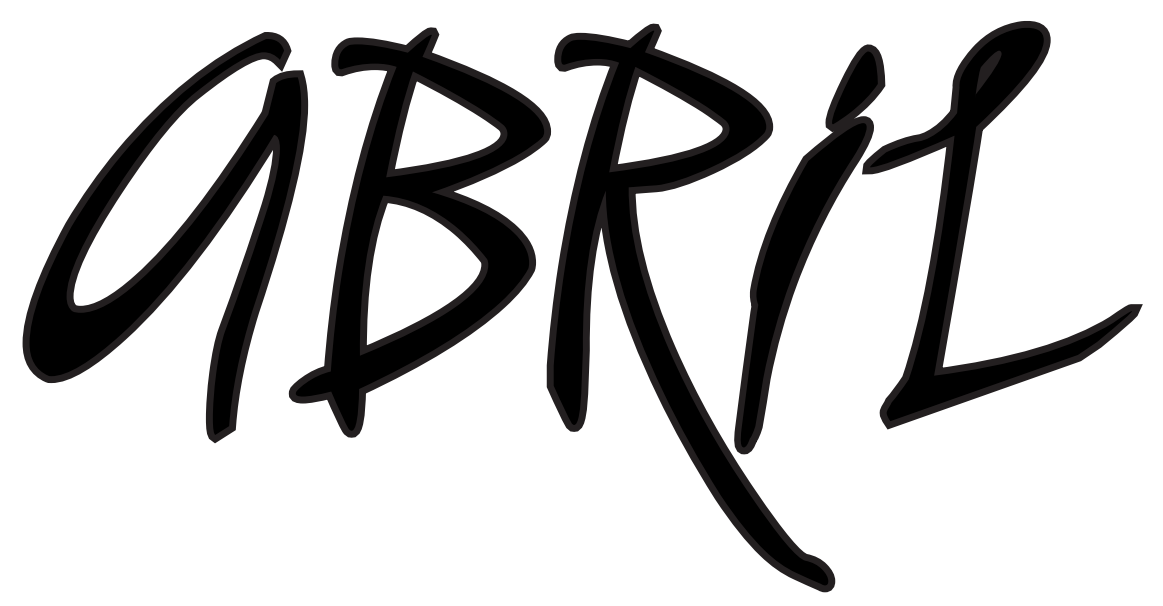

\section{NOVAS ESCRITURAS, NOVAS EPISTEMOLOGIAS}

Org.: Júlio Machado (UFF) e Nazir Can (UFRJ) 


\section{SUMÁRIO}

NOVAS ESCRITURAS, NOVAS EPISTEMOLOGIAS

APRESENTAÇÃO

.09

Júlio Machado e Nazir Can

O SOLAR, O CÉU E A NAÇÃO: CONTINUIDADES E RUPTURAS NAS LITERATURAS DE PAÍSES DE COLONIZAÇÃO PORTUGUESA

Maria Perla Araújo Morais e Frederico José Andries Lopesa

ENTRE O VENENO E O REMÉDIO, A LITERATURA: NARRATIVAS DA DOENÇA E DA CURA NA FICÇÃO DE MIA COUTO

José Welton Ferreira dos Santos Júnior e Rejane Vecchia da Rocha e Silva

BANTU SPIRITUALISM IN“O CESTO DE ADIVINHAÇÃO”BY ANA PAULA TAVARES

Vincenzo Cammarata

CHORIRO: A UTOPIA QUE SE FEZ VERBO

Adilson Fernando Franzin

PAISAGENS EMTRÂNSITO:UM RETORNO À CIDADE DE ULISSES

Francisca Marciely Alves Dantas e Maria Elvira Brito Campos

CONFLUÊNCIAS ENTRE MARGENS E ESPACIALIDADES EM LUCIANO SERAFIM E

GUIMARÃES ROSA . .93

Cristian de Oliveira Lopes e Debora Pereira Simões

A ENCENAÇÃO DA ETNOGRAFIA EM OS PAPÉIS DO INGLÊSENOVENOITES: ENTRE A NEGAÇÃO EOBOICOTE 109

Juliana Campos Alvernaz e Anita Martins Rodrigues de Moraes

APONTAMENTOS SOBRE ALTERIDADE E AUTOCTONIA NA FILOSOFIA AFRICANA: A PROPOSTA NEO-ANIMISTA DE RUY DUARTE DE CARVALHO

Christian Fischgold; Vanessa Riambau Pinheiro

"A INTESTINA BATALHA"SOCIALISTA MOÇAMBICANA ATRAVÉS DE CRÔNICA DA RUA 513.2, DE JOÃO PAULO BORGES COELHO

Fernanda Gallo 
LUÍS GONZAGA GOMES E A MACAU MITOLÓGICA: A AFIRMAÇÃO DE UMA IDENTIDADE SINGULAR

Fernando Ulisses Mendonça Serafim

PERFORMANDO IDENTIDADES DESTERRITORIALIZADAS: UMA LEITURA DE TRANS IBERIC LOVE, DE RAQUEL FREIRE 165

Marcelo Branquinho Massucatto Resende

MARCAS DA VIOLÊNCIA NO CONTO“STRESS", DE LÍLIA MOMPLÉ 181

Franciane Conceição da Silva

A REESCRITA DE MOÇAMBIQUE EM O ÚLTIMO VOO DO FLAMINGO, DE MIA COUTO 193

Thaíse Santana; Sirlei Santos Dudalski

NIKETCHE: UMA PERFORMANCE LITERÁRIA DE LIBERTAÇÃO 207

Renata Vaz Shimbo

FLUXOS IDENTITÁRIOS E AS RELAÇÕES DE PODER EM MOÇAMBIQUE 221

Daniela de Brito

NORMAS / EMENTAS

PRÓXIMAS EDIÇÕES 